\title{
Contingência e Contigüidade no Responder de Ratos Submetidos a Esquemas de Razão, Intervalo e Tempo Variáveis
}

\author{
Cristina Moreira Fonseca \\ Gerson Yukio Tomanari \\ Instituto de Psicologia, da Universidade de São Paulo
}

\begin{abstract}
RESUMO
No presente estudo, foram investigados os efeitos produzidos pela introdução de atraso do reforço sobre a taxa e a distribuição de freqüência das respostas de pressão à barra emitidas por oito ratos expostos a esquemas de intervalo, razão e tempo variáveis (VI, VR e VT, respectivamente). A partir de condições de linha de base sob reforçamento imediato, aplicou-se o atraso do reforço não sinalizado e non-resetting pelo tempo fixo de $5 \mathrm{~s}$ a cada um dos esquemas. Os resultados mostraram que, com a introdução do atraso do reforço, houve diminuição nas taxas de respostas de todos os sujeitos, em todos os três esquemas, mais acentuadamente sob VT, VI e VR, nesta ordem. A análise molecular dos dados revelou que, para os sujeitos sob VI, os valores efetivamente registrados de atrasos concentraram-se proximamente ao atraso programado de $5 \mathrm{~s}$, enquanto que, para os sujeitos expostos a VR, os intervalos assumiram valores menores. Esses resultados demonstram, portanto, que os efeitos do atraso do reforço sobre o responder operante variam em função do esquema de reforçamento vigente. No presente estudo, o uso de VI e VR, cujos controles pelo tempo e pelo número de respostas caracterizam, respectivamente, cada um desses esquemas, foi acompanhado por efeitos diferenciados tanto nas taxas de resposta quanto na distribuição do responder no tempo.
\end{abstract}

Palavras-chave: contingência; contigüidade; atraso do reforço; esquemas de reforçamento; ratos.

\section{ABSTRACT \\ Contingency and Contiguity on the Responding by Rats Exposed to Variable Interval, Variable Ratio and Variable Time Schedules of Reinforcement}

In this study, we examined the effects of introducing a delay of reinforcement on the rate and distribution of lever-pressing of eight rats under interval, ratio, and time reinforcement schedules (VI, VR, and VT). Following baseline conditions under immediate reinforcement, an unsignaled and nonresetting 5-s delay of reinforcement was introduced upon each of these schedules. Results showed that the delay was accompanied by a decrease in the rate of responses for all subjects, for all three schedules of reinforcement, most noticeable for VT, followed by the VI and then the VR schedules. A molecular analysis of the data revealed that, under VI, but not VR, the subjects' actual delay approximated the programmed value of 5 seconds. In general, results demonstrate that the effects of introducing a delay of reinforcement depend on the current reinforcement schedule. In the present study, in particular, response rate and response distribution under VI and VR, were followed by different effects of the delay when controlling respectively for time and ratio of responses.

Keywords: contingency; contiguity; delay of reinforcement; schedules of reinforcement; rats.

Na Análise do Comportamento, contingência e contigüidade são conceitos usados para se fazer referência a aspectos distintos de uma relação entre resposta e evento ambiental. No caso particular do comportamento operante, em que o comportamento é função de suas conseqüências, contingência refere-se a uma relação de dependência entre uma resposta e o evento ambiental subseqüente. Contigüidade, por sua vez, refere-se a uma relação puramente temporal entre os mesmos.
Em relações de contingência, o evento subseqüente (e conseqüente) não apenas acompanha temporalmente a resposta, mas é produzido por ela e sofre os seus efeitos retroativos. Nessas relações, a contigüidade entre a resposta e a sua conseqüência está presente em algum grau. Segundo dados experimentais fortemente estabelecidos na literatura, quanto menor o intervalo entre estes dois eventos, mais proeminentes são os efeitos da conseqüência sobre a resposta que a produziu (Catania, 1999; Keller \& Schoenfeld, 1950). 
Nesse sentido, a contigüidade entre uma resposta e a sua conseqüência exerceria um papel importante na relação de contingência. A medida desses efeitos, entretanto, ainda não é clara experimentalmente. Seria possível o estabelecimento e a manutenção de comportamentos sob condições em que vigorem exclusivamente relações de contigüidade? Eventos ambientais que se seguem a uma determinada classe de respostas, sem terem sido por elas produzidos, afetariam a probabilidade futura de ocorrência das respostas dessa classe?

Skinner (1948) expôs pombos privados de alimento a uma situação experimental na qual tinham acesso a comida em intervalos regulares de $15 \mathrm{~s}$, sem a exigência de qualquer resposta específica para isso. Mantidos nessa condição, o autor observou um aumento na freqüência de respostas que eram seguidas pela apresentação livre de comida. As topografias fortalecidas podiam ser diferentes para cada sujeito, mas, para a maioria deles, algum padrão era claramente constatado. Esse fenômeno passou a ser conhecido como um modelo de superstição comportamental, haja vista a demonstração de que seria possível o estabelecimento e a manutenção de repertórios comportamentais na ausência de uma relação de dependência entre resposta-conseqüência. Este trabalho de Skinner (1948), assim como outros que o seguiram destacando constatação semelhante (Critchfield \& Lattal, 1993; Dickinson, Watt, \& Griffiths, 1992; Lattal \& Gleeson, 1990; Snycerski, Laraway, Huitema, \& Poling, 2004; van Haaren, 1992; Wilkenfield, Nickel, Blakely, \& Poling, 1992), colocam em questão o papel da contingência (assim como o da contigüidade, em contrapartida) na complexa determinação do comportamento operante.

Diferentes estratégias metodológicas têm sido empregadas para abordar experimentalmente essa questão. Uma delas expõe os sujeitos experimentais a duas condições. Na primeira, respostas específicas produzem (imediatamente ou não) a apresentação de eventos conseqüentes. Na segunda, estes eventos são apresentados independentemente de qualquer resposta específica do organismo. Em situações como essa, os resultados gerais mostram que, relativamente ao responder inicial, a eliminação da contingência (mantida possível contigüidade) é seguida por uma diminuição na taxa de resposta (Catania \& Keller, 1981; Herrnstein, 1966; Lattal, 1972; Lattal \& Maxey, 1971; Sizemore \& Lattal, 1977; Williams, 1976; Zeiler, 1968).

Outra estratégia que tem sido largamente utilizada para avaliar o papel da contigüidade no responder operante tem como base os procedimentos chamados de atraso do reforço. Nestes, o tempo entre uma resposta e a conseqüência por ela produzida é manipulado e os efeitos desta manipulação, sobre o próprio responder, são analisados (Lattal, 1987).

Basicamente, o procedimento padrão de atraso do reforço emprega um esquema tandem ou encadeado (cf. Catania, 1999, que fornece maiores esclarecimentos sobre esses dois esquemas) no qual respostas no elo inicial que cumpram as exigências do esquema colocam em vigor o segundo elo. No segundo elo, vigora um esquema de tempo fixo ou variável, cujo valor determina o período de atraso do reforço.

Variações neste procedimento existem. Por exemplo, pode-se garantir que as respostas que produziram o reforço não possam ocorrer durante o período de atraso. Nesse caso, aplica-se uma contingência de DRO (Differential reinforcement of other behavior, cf. Catania, 1999) de modo que, a cada resposta emitida no segundo elo, reinicia-se o tempo de atraso (procedimento resetting). Nesse caso, o valor do atraso estabelecido pelo experimentador é necessariamente fixo (Azzi, Fix, Keller, \& Rocha e Silva, 1964; Dews, 1960; Lattal \& Ziegler, 1982; Pierce, Hanford, \& Zimmerman, 1972). Alternativamente ao procedimento resetting, o procedimento non-resetting permite que respostas possam ocorrer durante o período de atraso, sem alterá-lo. Nesse caso, a resposta que produziu o reforço não é necessariamente a precedente e, portanto, o valor do efetivo atraso pode não corresponder exatamente àquele programado pelo experimentador (Catania \& Keller, 1981; Dews, 1960; Gleeson \& Lattal, 1987; Lattal \& Ziegler, 1982; Morgan, 1970; Richards, 1981; Schneider, 1990; Sizemore \& Lattal, 1977, 1978; Williams, 1976). Finalmente, em ambas as variações de procedimento, resetting e nonresetting, o período de atraso pode ou não ser discriminado por um estímulo exteroceptivo; nesse caso, no lugar do esquema tandem, tem-se um esquema encadeado (Ferster, 1953; Richards, 1981).

No conjunto, em se tratando particularmente dos estudos experimentais com infra-humanos, os resultados dos trabalhos nesta área demonstram que, em geral, os atrasos do reforço diminuem as taxas de respostas de pombos e ratos em relação à linha de base a despeito do tipo de procedimento usado (Azzi \& cols., 1964; Catania \& Keller, 1981; Richards, 1981; Sizemore \& Lattal, 1977, 1978; Williams, 1976). Essa diminuição reflete uma função inversa do valor do atraso, ou seja, quanto maior o atraso, menor a taxa de resposta que o acompanha. Comparando-se os efeitos 
gerados por atrasos sinalizados e não-sinalizados, os dados mostram que taxas de respostas mais elevadas tendem a ser mantidas, mesmo sob valores altos de atraso, quando estes são sinalizados (Dews, 1960; Ferster, 1953).

A despeito dos resultados relativamente consistentes que têm gerado, os procedimentos que investigam o papel do atraso do reforço são passíveis de crítica. Nesse sentido, é evidente o fato de que o procedimento resetting, para manter fixo o valor do atraso, introduz explicitamente uma contingência (DRO) que, em si, reduz a freqüência do responder. Portanto, a diminuição na taxa de respostas que acompanha os atrasos do reforço deriva-se, não apenas do atraso, mas também do próprio DRO. Dessa forma, ao garantir o valor fixo do atraso, o procedimento acrescenta, à complexidade do fenômeno estudado, condições que dificultam ainda mais a possibilidade de se chegar a conclusões específicas e claras com relação à variável central em análise, isto é, o atraso do reforço.

Em vista dessa constatação, o procedimento nonresetting mostra-se mais apropriado para a investigação dos efeitos do atraso do reforço. No entanto, este procedimento, ao permitir que ocorram respostas durante o período programado de atraso, torna o efetivo atraso dependente do responder, variável e geralmente menor do que o programado. Em outras palavras, o atraso é tomado como uma variável independente, cujos valores efetivamente obtidos dependem do responder do próprio animal e, portanto, das contingências em vigor.

Metodologicamente, para acompanhar os efeitos de uma variável independente com estas características altamente dinâmicas em que cada atraso assume um valor particular, é necessário contar com equipamentos que permitam o devido controle das contingências e grande precisão de registro. Condições assim eram difíceis de ser obtidas quando se dispunham apenas de equipamentos eletromecânicos. Entretanto, nas atuais condições computacionais, tais dificuldades foram contornadas de modo que, no caso particular dos estudos sobre atraso do reforço, pode-se facilmente trabalhar com os reais valores de cada atraso obtido na sessão experimental, conforme sugerido por Schneider (1990).

Assim, a partir dessa atual conjuntura metodológica, o presente estudo teve por objetivo retomar o "atraso do reforço" como questão experimental e investigar os efeitos produzidos pela introdução do atraso programado do reforço, no caso, de $5 \mathrm{~s}$, não sinali- zado e non-resetting, sobre a taxa e a distribuição de freqüência no tempo de respostas de pressão à barra emitidas por ratos submetidos a diferentes esquemas de intervalo, razão e tempo variáveis, haja vista os diferentes padrões típicos gerados por cada um desses esquemas.

\section{MÉTODO}

\section{Sujeitos}

Foram sujeitos deste trabalho oito ratos ingênuos, albinos (Ratttus novergicus), Wistar, machos. No início do experimento, os sujeitos tinham entre 100 e 110 dias de vida. Alojados em gaiolas individuais, eram mantidos a $85 \%$ do peso ad libitum por regime de restrição de acesso à água. Alimento estava constantemente disponível.

\section{Equipamento}

Foram usadas quatro caixas de condicionamento operante modulares, modelo ENV-007, fabricadas por Med Associates Inc. As caixas estavam equipadas com um bebedouro localizado no centro da parede direita. Em repouso, o bebedouro era mantido mergulhado em uma cuba d'água localizada na parte externa da caixa. A cada acionamento do bebedouro, uma concha com cerca de 0,06 ml de água era introduzida na caixa, onde permanecia por $4 \mathrm{~s}$. À direita do bebedouro encontrava-se uma barra de respostas. Localizada na parede oposta ao bebedouro, havia uma lâmpada que produzia a iluminação ambiente durante a sessão experimental.

As sessões experimentais foram controladas por um micro-computador compatível com IBM-PC, uma interface (DIG-700P1) e o aplicativo Windows MedPC (SOF-735), os quais, a partir de rotinas de programação desenvolvidas no programa Turbo Pascal v. 1.5, em linguagem MedState Notation, comandaram as contingências nas caixas experimentais e registraram as respostas de pressão à barra.

\section{Procedimento}

Inicialmente, todos os oito sujeitos passaram por um treino preliminar que envolveu o treino ao bebedouro, a modelagem da resposta de pressão à barra e o fortalecimento dessa resposta em esquema de reforçamento contínuo, nessa ordem. Na seqüência, as respostas de pressão à barra foram reforçadas em esquemas intermitentes e, para isso, os sujeitos foram distribuídos em dois grupos em função dos esquemas a que seriam expostos posteriormente (Grupo VI e 
Grupo VR). A partir do reforçamento contínuo, os sujeitos do Grupo VI foram submetidos diretamente a VI 20 s. Os sujeitos do Grupo VR, diferentemente, foram submetidos a razões aumentadas progressivamente ao longo das sessões (VR 3, VR 6, VR 10 e VR 15).

Durante o treino inicial, bem como ao longo de todas as fases experimentais, as sessões eram encerradas após a liberação de 100 gotas de água. Este treino inicial foi mantido até que os sujeitos dos Grupos VI e VR apresentassem estabilidade na taxa de respostas em, respectivamente, VI 20 s e VR 15, com variações menores do que cerca de $10 \%$ em três sessões consecutivas, avaliadas por inspeção visual. O mesmo critério de estabilidade foi aplicado para mudanças de fase ao longo das quatro fases que compuseram o experimento (Tabela 1).

TABELA 1

Distribuição dos Sujeitos nos Grupos VI e VR ao Longo das Quatro Fases Experimentais

\begin{tabular}{lllll}
\hline \multirow{2}{*}{ Ratos } & \multicolumn{1}{c}{ Fases } & \multicolumn{1}{c}{3} & \multicolumn{1}{c}{4} \\
\cline { 2 - 5 } Grupo VI & \multicolumn{1}{c}{2} & & \\
\hline 149 & VI 20 & tand VI 20 FT 5 & VI 20 & VT 20 \\
150 & VI 20 & tand VI 20 FT 5 & VI 20 & VT 20 \\
151 & VI 20 & VT 20 & VI 20 & tand VI 20 FT 5 \\
152 & VI 20 & VT 20 & VI 20 & tand VI 20 FT 5 \\
\hline Grupo VR & & & VR 15 & VT 20 \\
\hline 153 & VR 15 & tand VR 15 FT 5 & VR 15 & VT 20 \\
154 & VR 15 & tand VR 15 FT 5 & VR 15 & tand VR 15 FT 5 \\
155 & VR 15 & VT 20 & VR 15 & tand VR 15 FT 5 \\
156 & VR 15 & VT 20 & & \\
\hline
\end{tabular}

\section{Grupo VI}

A partir de fases de linha de base em VI $20 \mathrm{~s}$ (Fases 1 e 3), seguiram-se as fases em tandem VI 20 s FT 5 s (Fase 2) e VT 20 s (Fase 4), para os Ratos 149 e 150. Para os Ratos 151 e 152, a ordem das Fases 2 e 4 foi invertida.

Sob VI 20 s, um reforço era liberado contingentemente à passagem de um intervalo de tempo médio programado de $20 \mathrm{~s}$, seguido pela emissão de uma resposta de pressão à barra. Os valores dos intervalos entre reforços programados para o VI foram: 2, 4, 6, $8,10,12,14,16,18,20,22,24,26,28,30,32,34,36$ e 38 s. Cada intervalo repetiu-se cinco vezes em cada sessão, com exceção do intervalo de 20 s, que se repetiu dez vezes.

Sob tandem VI 20 s FT 5 s, assim que completada a exigência do intervalo corrente do esquema de VI 20 $\mathrm{s}$ (programado exatamente como descrito acima), iniciava-se o tempo de $5 \mathrm{~s}$ (atraso do reforço), ao fim do qual uma gota de água era liberada independentemente de qualquer resposta do sujeito. Respostas durante o FT não tinham conseqüências programadas, porém eram registradas.

Sob VT 20 s, uma gota de água era liberada após transcorrido um tempo médio de $20 \mathrm{~s}$, a despeito de qualquer resposta do sujeito. Os períodos entre possíveis respostas e a liberação de água foram registrados. No VT, foram utilizados os mesmos intervalos variáveis programados para o esquema VI $20 \mathrm{~s}$ e acima identificados.

\section{Grupo VR}

A partir de fases de linha de base em VR 15 (Fases 1 e 3), seguiram-se fases em tandem VR 15 FT $5 \mathrm{~s}$ (Fase 2) e VT 20 s (Fase 4), para os Ratos 153 e 154. Para os Ratos 155 e 156, a ordem das Fases 2 e 4 foi invertida.

Sob VR 15, uma gota de água era liberada contingentemente à emissão de um número médio programado de 15 respostas de pressão à barra. Os valores das razões programadas para o VR foram: 1, 3, 5, 7, 9, $11,13,15,17,19,21,23,25,27$ e 29 . Cada razão se repetiu sete vezes em cada sessão, com exceção das razões 1, 5, 9, 15 e 27, que se repetiram seis vezes.

Sob tandem VR 15 FT 5 s, assim que completada a exigência do intervalo corrente do VR 15 (programado exatamente como descrito acima), iniciava-se o tempo de $5 \mathrm{~s}$ (atraso do reforço), ao fim do qual uma gota de água era liberada. Respostas durante o FT não tinham conseqüências programadas, porém eram registradas. 
Para os sujeitos do Grupo VR, a programação do VT 20 s foi mantida exatamente como descrita acima para os sujeitos do Grupo VI.

\section{RESULTADOS}

A Figura 1 fornece uma análise geral dos dados ao mostrar as taxas de respostas de pressão à barra dos Grupos VI (Ratos 149, 150, 151 e 152; Painel A) e
VR (Ratos 153, 154, 155 e 156; Painel B), nas últimas seis sessões de cada uma das fases de linha de base, nas seis primeiras e seis últimas sessões sob atraso do reforço (esquema tandem) e nas seis primeiras e seis últimas sessões sob VT 20 s. Para cada dupla de sujeito, uma seqüência diferente de fases foi seguida.

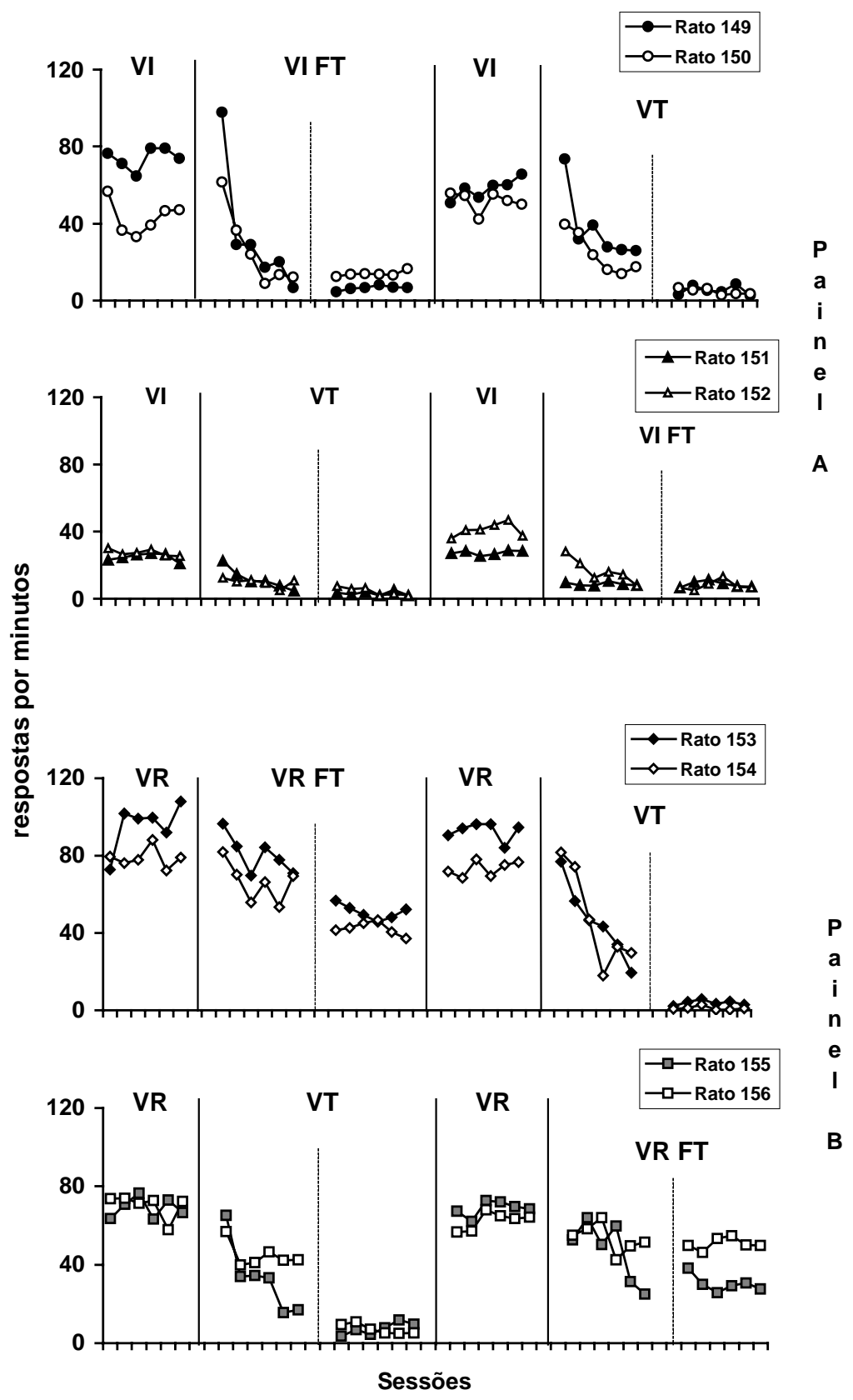

Figura 1. Taxa de resposta de pressão à barra dos sujeitos do Grupo VI (Painel A) e do Grupo VR (Painel B) nas seis últimas sessões em linha de base inicial e retorno à linha de base e, nas seis primeiras e seis últimas sessões com atraso de $5 \mathrm{~s}$ e sob VT $20 \mathrm{~s}$. Na figura, as linhas sólidas separam as diferentes condições e as linhas tracejadas separam as seis primeiras das seis últimas sessões da mesma fase. 
Em linha de base, as taxas de respostas nas últimas seis sessões diferiram entre os sujeitos, porém, a todos, observa-se relativa estabilidade ao longo das sessões. Para os Ratos 151 e 152, sob VI, as taxas de respostas mantiveram-se em torno de 30 respostas/minuto (resp/min). Para os Ratos 149 e 150, também sob VI, estas estiveram entre $40 \mathrm{resp} / \mathrm{min}$ e 80 $\mathrm{resp} / \mathrm{min}$. Para os quatro animais sob VR, as taxas mantiveram-se próximas a $80 \mathrm{resp} / \mathrm{min}$. De modo geral, portanto, o esquema de VR 15 gerou taxas mais elevadas em linha de base do que o esquema de VI 20 s. Comparando-se as duas fases de linha de base entre si, observa-se que, para todos os sujeitos, em geral, a segunda exposição ao VR e ao VI é acompanhada da recuperação do desempenho obtido na primeira exposição. Nesse aspecto, os dados dos Ratos 149 e 152, em particular, mostram uma pequena diminuição e um pequeno aumento, respectivamente, na taxa de resposta na segunda condição de linha de base relativamente à primeira.

A introdução do atraso de reforço, para todos os sujeitos, em ambos os esquemas, foi acompanhada de uma acentuada diminuição da taxa de resposta relativamente à linha de base. Para os animais expostos ao VI, ao final da sexta sessão, as taxas de respostas haviam praticamente atingido os valores estáveis registrados ao longo das seis últimas sessões, ou seja, entre $6 \mathrm{resp} / \mathrm{min}$ e $14 \mathrm{resp} / \mathrm{min}$. Para os animais sob VR, ao final da fase com atraso de reforço, as taxas mantiveram-se entre $30 \mathrm{resp} / \mathrm{min}$ e $50 \mathrm{resp} / \mathrm{min}$.

Independentemente da ordem de apresentação das fases sob os esquemas com atraso de reforço (tandem) ou apresentação do reforço independentemente de resposta (VT), ambos produziram efeitos semelhantes sobre as quedas nas taxas de respostas em relação ao responder em linha de base. Sob VT, entretanto, as taxas de respostas mantiveram-se baixas para todos os sujeitos, em torno de $10 \mathrm{resp} / \mathrm{min}$ ou menos.

Os efeitos do atraso do reforço sob VI e VR, além do VT, devem ainda ser avaliados em termos relativos à respectiva linha de base de cada sujeito individualmente. Nesse caso, tomando-se as taxas médias de respostas nas seis últimas sessões, é possível constatar que, para os sujeitos sob VI, estas se mantiveram entre cerca de $10 \%$ e $30 \%$ dos valores da linha de base. Para os sujeitos sob VR, a taxa de resposta esteve entre cerca de $40 \%$ e $80 \%$. Sob VT, considerando-se todos os oito sujeitos, a taxa de resposta diminuiu um pouco mais acentuadamente, estabelecendo-se entre cerca de $3 \%$ e $10 \%$. Relativamente às respectivas linhas de base, portanto, a introdução do atraso do reforço foi acompanhada por uma diminuição mais acentuada no responder sob VI do que sob VR. O responder sob $\mathrm{VT}$, tanto nas sessões iniciais quanto nas sessões finais dessa fase, assemelhou-se ao responder sob o esquema de VI, exceto pelo fato de que, em condições estáveis, o VT foi acompanhado, em geral, por taxas de respostas ainda menores.

Em decorrência de o procedimento empregar um atraso non-resetting, os intervalos efetivamente obtidos entre a apresentação do reforço e a última resposta que o precede são variáveis e dependem do próprio responder do animal. Assim, a Figura 2 mostra a distribuição de freqüência dos valores de atrasos efetivamente registrados segundo a média obtida nas seis últimas sessões sob VI (gráficos à esquerda) e VR (gráficos à direita). 
Grupo VI
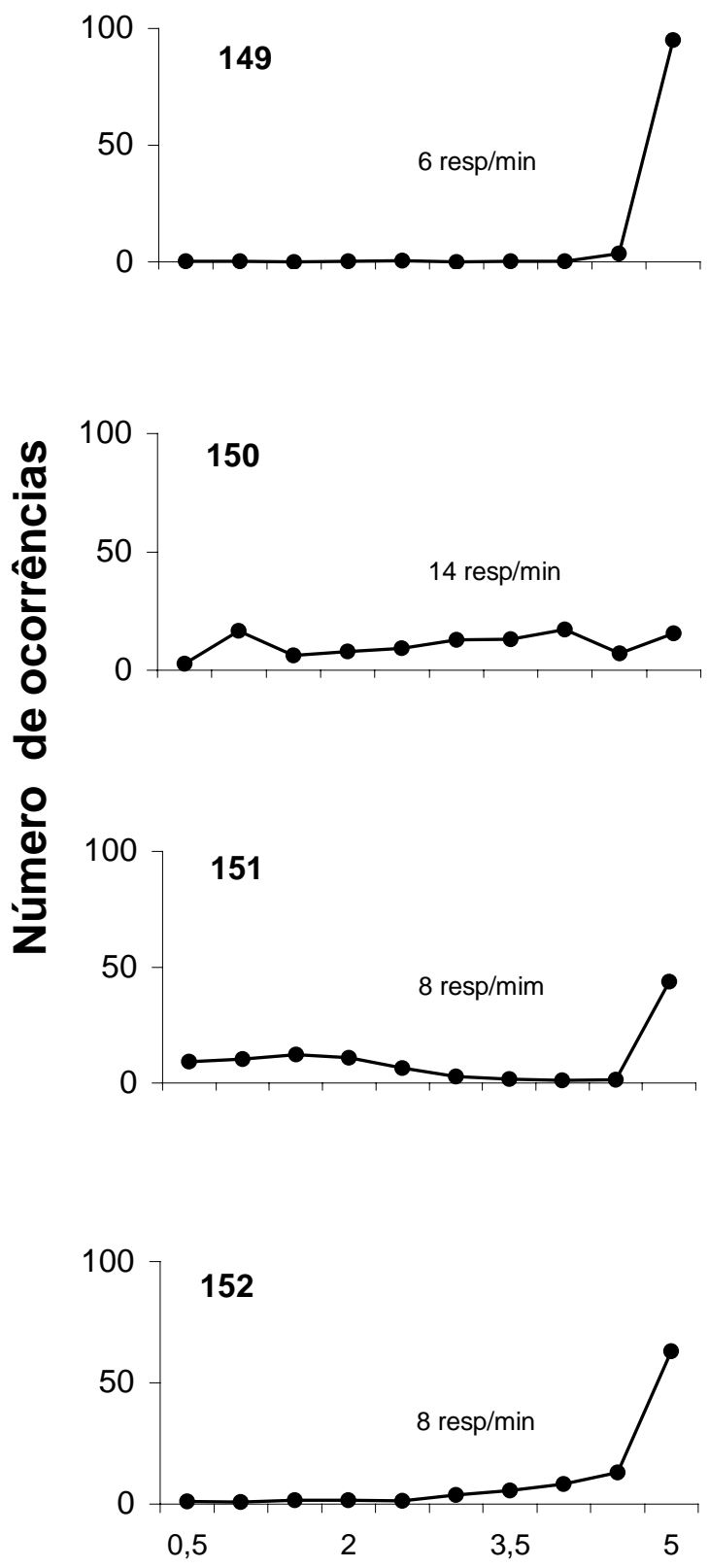

Grupo VR

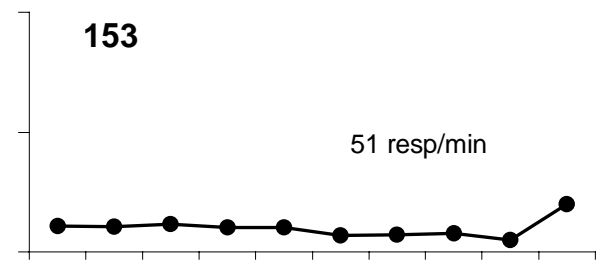

154

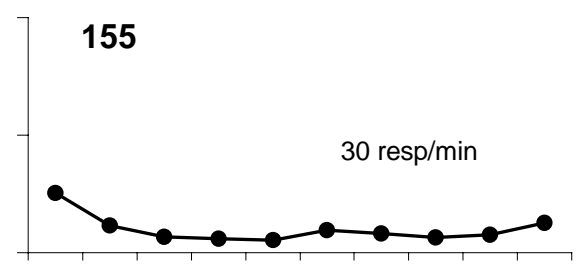

156

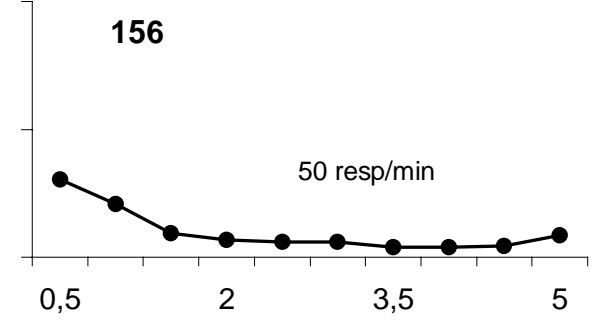

atrasos obtidos (segundos)

Figura 2. Distribuição de freqüência dos valores de atrasos registrados segundo a média obtida nas últimas seis sessões sob atraso do reforço para os sujeitos do Grupo VI (gráficos à esquerda na figura) e do Grupo VR (gráficos à direita). A taxa média de resposta nas seis últimas sessões desta fase acompanha cada distribuição.

Por esta análise, podem-se verificar efeitos claramente diferenciados do atraso sob o responder em VI e VR. Para os sujeitos sob VI, os valores de atrasos efetivamente registrados concentraram-se proximamente aos valores do atraso programado de $5 \mathrm{~s}$, com exceção do Rato 150, cujos atrasos obtidos foram mais igualmente distribuídos ao longo dos $5 \mathrm{~s}$ máximos de atraso. Para os sujeitos sob VR, os valores de atrasos registrados concentraram-se entre $0,5 \mathrm{~s}$ e $1,0 \mathrm{~s}$. Para o Rato 153, diferentemente dos demais, os valo- 
res de atrasos distribuíram-se de modo a se concentrarem entre 4,5 s e 5,0 s. De modo geral, portanto, os atrasos do reforço efetivamente obtidos sob VI e VR diferiram, sendo maiores no primeiro esquema. Esta análise complementa a anterior e permite compreender as reduções mais acentuadas nas taxas de respostas em VI do que em VR.

Os intervalos em que vigoravam os atrasos do reforço não eram sinalizados no presente procedimento. Portanto, os intervalos entre respostas (IRTs) nas diferentes fases experimentais poderiam refletir, de modo geral, os próprios efeitos dos esquemas em vigor.
Procedendo-se com essa análise, as Figuras 3 e 4 mostram a distribuição de IRTs segundo a média obtida nas seis últimas sessões de cada fase a que foram submetidos os sujeitos sob VI (Figura 3) e sob VR (Figura 4). Em ambas as figuras, as duas primeiras linhas de gráficos referem-se às fases de linhas de base. A terceira linha refere-se à fase em que vigorou o atraso de reforço. A última linha refere-se à fase em que vigorou o esquema de VT. A disposição dos gráficos nessas duas figuras, portanto, não corresponde à seqüência de fases experimentais.

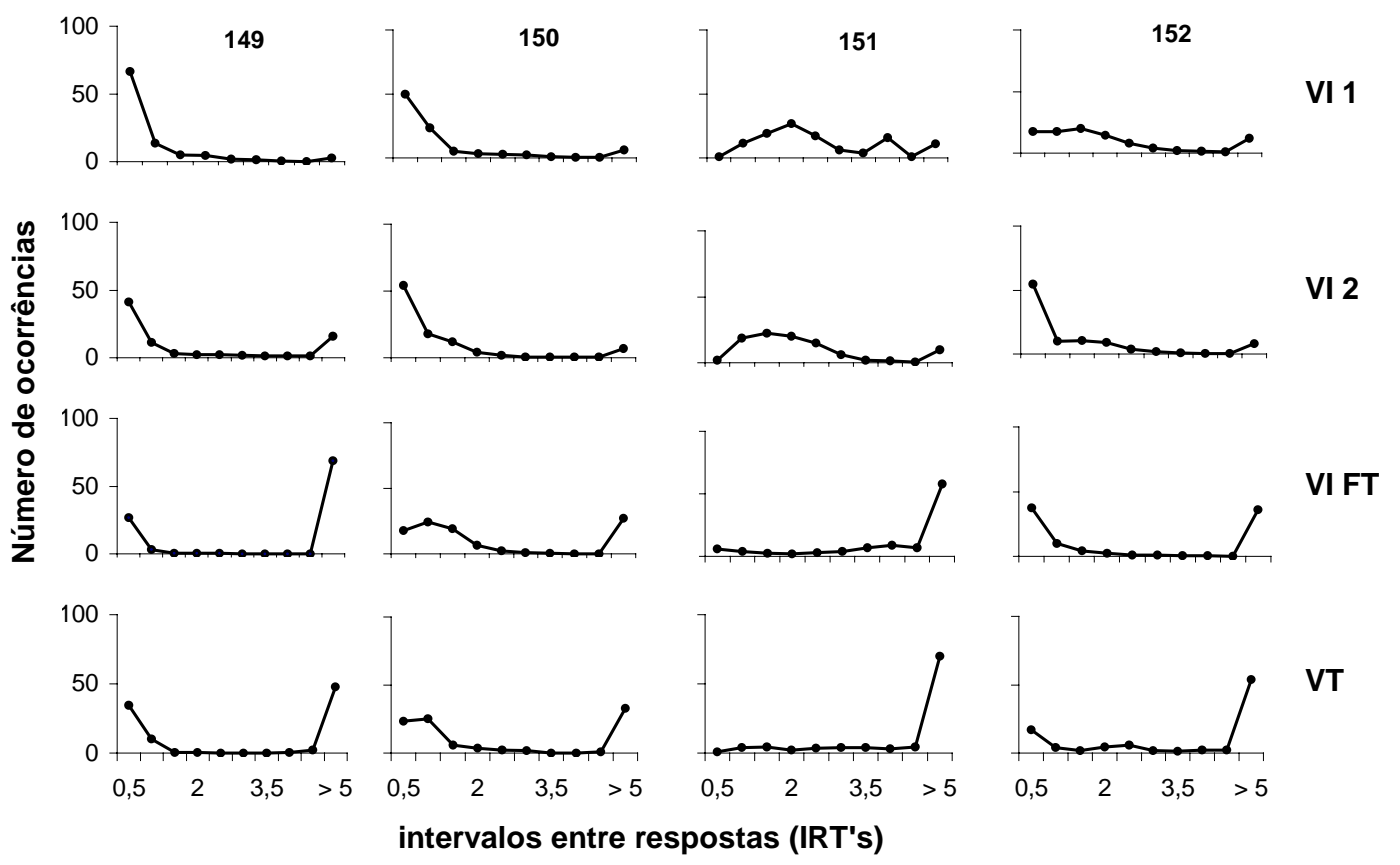

Figura 3. Distribuição de freqüência de intervalos entre respostas (IRTs) registrados segundo a média obtida nas últimas seis sessões de cada fase para os sujeitos do Grupo VI. Na Figura, as fases são apresentadas na seguinte ordem: VI 1 e VI 2 (linha de base e retorno à linha de base), tand VI FT e VT.

A partir da Figura 3, é possível observar que, em linha de base sob VI, os IRTs mais freqüentes concentraram-se entre 0,5 e 2,0 s. Na vigência do atraso do reforço ou VT, diferentemente, os IRTs que passaram a predominar eram maiores do que 5,0 s. 


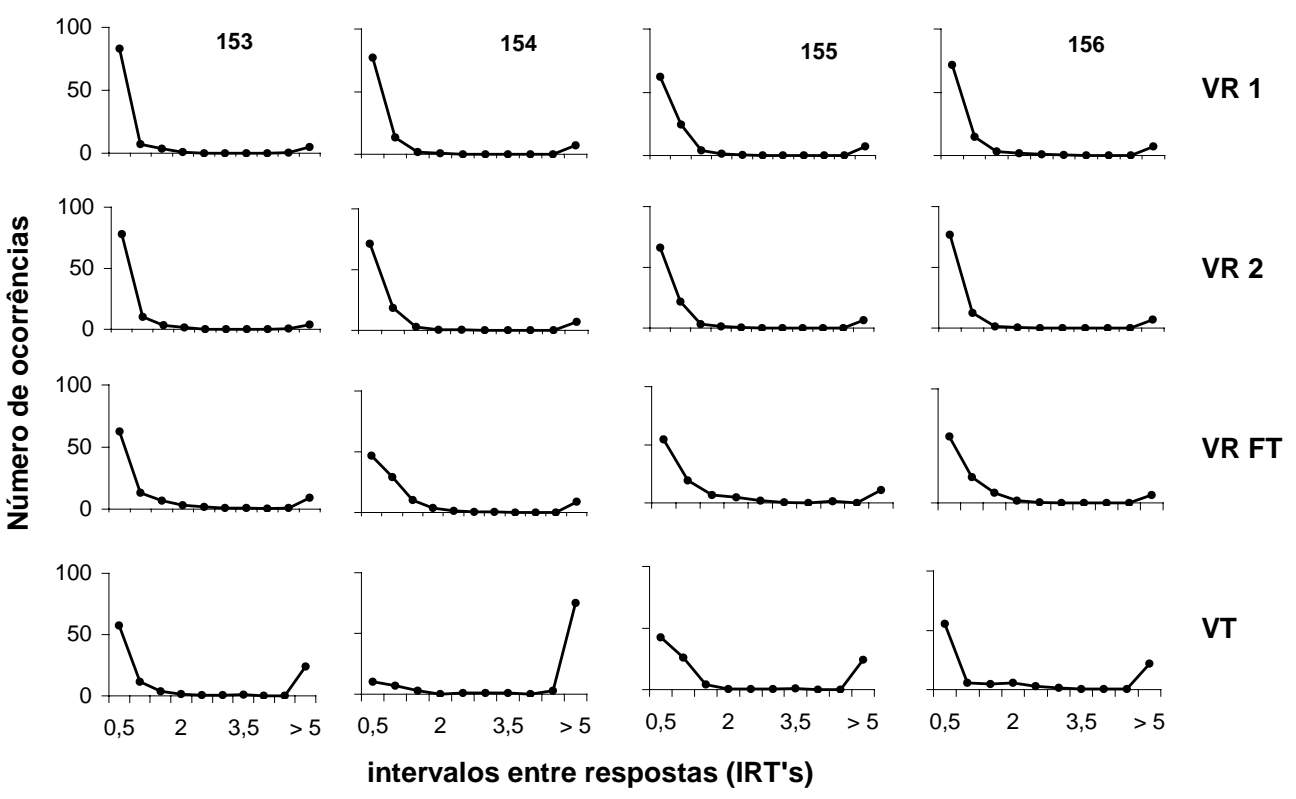

Figura 4. Distribuição de freqüência de intervalos entre as respostas (IRTs) registrados segundo a média obtida nas últimas seis sessões de cada fase para os sujeitos do Grupo VR. Na Figura, as fases são apresentadas na seguinte ordem: VR 1 e VR 2 (linha de base e retorno à linha de base), tand VR FT e VT.

Observando-se a Figura 4, por outro lado, verificase que, sob VR, IRTs freqüentes entre 0,5 e $1,0 \mathrm{~s}$ ocorreram tanto nas fases de linha de base quanto nas fases com atraso do reforço. Sob VT, interessantemente, além de se concentrarem nos intervalos entre 0,5 e $1 \mathrm{~s}$, os IRTs concentraram-se também nos intervalos maiores do que 5,0 s, com exceção dos dados do Rato 154, que demonstram somente os IRTs maiores.

Comparando-se as Figuras 3 e 4, portanto, é possível verificar que a introdução do atraso do reforço alterou mais claramente os intervalos entre as respostas sob VI do que sob VR, no sentido de aumentá-los. Efeito semelhante ocorreu sob VT, ainda que, para os sujeitos com história em VR, intervalos curtos entre respostas ainda se mantiveram em freqüência destacável.

\section{DISCUSSÃO}

Os dados do presente estudo mostram que os efeitos da introdução do atraso do reforço sobre o responder operante interagem com os esquemas de reforçamento em vigor e produzem resultados diferenciados a cada um deles. Em diferentes e complementares análises de dados, foram comparados os esquemas de VI 20 s e VR 15, aos quais se introduziu um atraso fixo non-resetting e não sinalizado de $5 \mathrm{~s}$.

Basicamente, verificou-se que a introdução do atraso, dentro dos parâmetros empregados, foi acom- panhado por uma clara e imediata redução nas taxas de respostas. Em VI, esta diminuição foi mais acentuada do que em VR, fato que nos convoca a uma análise detalhada das variáveis controladoras do responder. Na ausência do atraso, o esquema de VI 20 s estabelecia a liberação de um reforço mediante a passagem de um intervalo variável de 2 a 38 s, após o qual deveria ocorrer uma resposta de pressão à barra. $\mathrm{O}$ atraso de reforço de $5 \mathrm{~s}$ foi acrescentado a esses intervalos e, com isso, produziu automaticamente um aumento na distribuição temporal dos reforços ao longo da sessão (Lattal, 1987). Por exemplo, o menor intervalo entre reforços passou de $2 \mathrm{~s}$ para $7 \mathrm{~s}$ e o maior intervalo entre reforços passou de $38 \mathrm{~s}$ para $43 \mathrm{~s}$. Além disso, considerando-se que o tempo programado para a liberação de cada um dos reforços aumentou em $5 \mathrm{~s}$, a vigência do atraso implicou um aumento no tempo total da sessão em, pelo menos, 8 min.

Sob VR 15, um reforço era liberado contingentemente à emissão de uma quantidade variável de 1 a 29 respostas, sem qualquer requisito temporal préestabelecido. Com a introdução do atraso, um requisito temporal foi adicionado ao VR, esquema não definido por parâmetros temporais. Assim, considerando-se que as respostas durante o atraso não o afetavam, tampouco podiam estar sob controle discriminativo, a introdução do atraso resultou em um au- 
mento no número de respostas, ainda que este aumento não fizesse parte da razão exigida.

Em termos dos aspectos controladores do responder, a introdução do atraso modificou tão somente dimensões temporais do esquema de VI. Diferentemente, em se tratando do VR, parâmetros temporais do atraso foram introduzidos a um esquema de linha de base em que a quantidade de respostas era o critério para reforçamento. Nesse caso, a um requisito quantitativo, acrescentou-se um requisito de dimensão temporal inexistente até então na história experimental daqueles sujeitos.

Os efeitos da linearidade ou não nas dimensões controladoras do responder sob atraso aplicado a VI e VR, respectivamente, podem ter contribuído para os resultados presentes. A partir da análise que abordou os reais valores dos atrasos obtidos sob cada esquema, verificou-se que, de fato, os maiores atrasos do reforço, aqueles que mais se aproximavam do valor do atraso programado de $5 \mathrm{~s}$, estavam sob o responder em VI. O responder em VR, ao contrário, foi acompanhado por atrasos claramente mais curtos. Ou seja, os atrasos em cada esquema assumiram valores diferentes e compatíveis com as taxas de respostas gerais obtidas, no sentido de que, quanto maior o valor do atraso, menor as taxas de respostas que o acompanham, e vice-versa.

A diminuição na freqüência das respostas sob efeito do atraso do reforço pode ter favorecido o fortalecimento de outras respostas, diferentes do pressionar a barra, que tenham ocorrido temporalmente próximas à liberação do reforço. Nesse caso, a freqüência dessas outras respostas pode ter aumentado, competido com as respostas de pressão à barra e, assim, contribuído para que diminuíssem em freqüência. Essa análise, formulada por Richards (1981), parece condizente com os resultados obtidos no presente estudo. Para os sujeitos expostos ao VI, esquema em que vigoram requisitos fundamentalmente temporais para o reforçamento, foi justamente uma diminuição como essa, sobre as respostas de pressão à barra, que se verificou. Para os sujeitos submetidos ao VR, em contrapartida, no qual um conjunto (ou jorro) de respostas é reforçado e, portanto, outros comportamentos são menos prováveis de serem emitidos e reforçados, as taxas de pressões à barra foram menos afetadas pelo atraso.

No presente procedimento, o período de atraso do reforço não foi sinalizado. Por essa razão, os efeitos do atraso puderam ser analisados por meio da distribuição dos intervalos entre respostas (IRTs) nas ses- sões das diferentes condições experimentais. Nesse caso, reiterando-se os dados anteriores, foi possível verificar que a introdução do atraso alterou os IRTs no sentido de aumentá-los, tanto na vigência do atraso quanto na vigência do VT. Os efeitos sobre o responder em VR, entretanto, foram distintos, não alterando os IRTs. Interessantemente, ratos com a história em VR, quando submetidos ao VT, mantiveram uma freqüência observável de IRTs curtos, mesmo quando as taxas de respostas já haviam se reduzido em até $90 \%$ daquelas presentes em linha de base.

A utilização do esquema de VT forneceu, no presente trabalho, parâmetros do responder na ausência de relações de contingência, ainda que na presença possível de relações de contigüidade. De forma contrabalanceada entre os sujeitos, essa condição foi estrategicamente inserida antes ou depois do contato dos sujeitos com a contingência de atraso do reforço. Efeitos de uma história experimental como essa, no presente estudo, não evidenciou qualquer efeito aparente.

O esquema de VT estabelece a liberação de uma gota de água transcorrido um intervalo médio de $20 \mathrm{~s}$, a despeito de qualquer resposta do sujeito. Havendo-se pressões à barra sob controle da apresentação de água, o controle das mesmas deve ser fundamentalmente temporal. Por essa razão, possivelmente, as taxas de respostas sob VT, seguindo-se às linhas de base em VI ou VR, diminuem à semelhança do desempenho em VI com atraso, ainda que um pouco mais acentuadamente. A partir de uma análise feita estritamente com base nas taxas de respostas, portanto, são efeitos muito pouco claros aqueles que decorrem da contingência de VI com atraso de reforço (e não de VR com atraso) e a série de contigüidades resposta/água promovida pelo VT. Investigações futuras que manipulem parametricamente os valores utilizados presentemente poderão contribuir para aprofundar e generalizar o papel relativo das relações de contingência e contigüidade na manutenção de uma resposta operante pelo evento que a segue. Ao que os dados aqui relatados indicam, a expressão comportamental dessas relações podem muitas vezes ser praticamente indiferenciadas a depender do esquema vigente.

\section{REFERÊNCIAS}

Azzi, R., Fix, D. S. R., Keller, F. S., \& Rocha e Silva, M. I. (1964). Exteroceptive control of response under delayed reinforcement. Journal of the Experimental Analysis of Behavior, 7, 159-162.

mento, linguagem e cognição. (Deisy das Graças de Souza, Trad.). Porto Alegre: Artes Médicas. 
Catania, A. C., \& Keller, K. J. (1981). Contingency, contiguity, correlation, and the concept of causation. Em P. Harzem, \& M. D. Zeiler (Orgs.), Predictability, correlation, and contiguity (pp. 125-167). London: Wiley.

Critchfield, T. S., \& Lattal, K. A. (1993). Acquisition of a spatially defined operant with delay reinforcement. Journal of the Experimental AnalysiCatania, A. C. (1999). Aprendizagem: Comportas of Behavior, 59, 373-387.

Dews, P. B. (1960). Free-operant behavior under conditions of delayed reinforcement. I. CRF-type schedules. Journal of the Experimental Analysis of Behavior, 3, 221-234.

Dickinson, A., Watt, A., \& Griffiths, W. J. H. (1992). Freeoperant acquisition with delayed reinforcement. The Quarterly Journal of Experimental Psychology, 45B, 241-258.

Ferster, C. B. (1953). Sustained behavior under delayed reinforcement. Journal of Experimental Psychology, 45, 218-224.

Gleeson, S., \& Lattal, K. A. (1987). Response-reinforcer relations and the maintenance of behavior. Journal of the Experimental Analysis of Behavior, 48, 383-393.

Herrnstein, R. J. (1966). Superstition: A corollary of the principles of operant conditioning. Em W. K. Honig (Org.), Operant behavior: Areas of research and application (pp. 33-51). New York: Appleton-Century-Crofts.

Keller, F. S., \& Schoenfeld, W. N. (1950). Principles of psychology. New York: AppletonCentury-Crofts.

Lattal, K. A. (1972). Response-reinforcer independence and conventional extinction after fixed-interval and variable-interval schedules. Journal of the Experimental Analysis of Behavior, 18, 133-140.

Lattal, K. A. (1987). Considerations in the experimental analysis of reinforcement delay. Em M. L. Commons, J. E. Mazur, J. A. Nevin, \& H. Rachlin (Orgs.), Quantitative analyses of behavior: Vol. 5. The effect of delay and of intervening events on reinforcement value (pp.107-123). Hillsdale, NJ: Erlbaum.

Lattal, K. A., \& Gleeson, S. (1990). Response acquisition with delay reinforcement. Journal of Experimental Psychology: Animal Behavior Process, 16, 27-39.

Lattal, K. A., \& Maxey, G. C. (1971). Some effects of responseindependent reinforcers in multiple schedules. Journal of the Experimental Analysis of Behavior, 16, 225-231.

Lattal, K. A., \& Ziegler, D. R. (1982). Briefly delayed reinforcement: An interresponse time analysis. Journal of the Experimental Analysis of Behavior, 37, 407-416.
Morgan, M. J. (1970) Fixed-interval schedules and delay of reinforcement. Quarterly of Experimental Psychology, 22, 663673.

Pierce, C. H., Handford, P. V, \& Zimmerman, J. (1972). Effects of different delay of reinforcement procedures on variableinterval responding. Journal of the Experimental Analysis of Behavior, 18, 141-146.

Richards, R. W. (1981). A comparison of signaled and unsignaled delay of reinforcement. Journal of the Experimental Analysis of Behavior, 35, 145-152.

Schneider, S. M. (1990). The role of contiguity in free-operant unsignaled delay of positive reinforcement: A brief review. The Psychological Record, 40, 239-257.

Sizemore, O. J., \& Lattal, K. A. (1977). Dependency, temporal contiguity, and response-independent reinforcement. Journal of the Experimental Analysis of Behavior, 25, 119-125.

Sizemore, O. J., \& Lattal, K. A. (1978). Unsignaled delay of reinforcement in variable-interval schedules. Journal of the Experimental Analysis of Behavior, 30, 169-175.

Skinner, B. F. (1948). 'Superstition' in the pigeon. Journal of Experimental Psychology, 38,168-172.

Snycerski, S., Laraway S., Huitema, B. E., \& Poling, A. D. (2004). The effects of behavioral history on response acquisition with immediate and delayed reinforcement. Journal of the Experimental Analysis of Behavior, 81, 51-64.

Van Haaren, F. (1992). Response acquisition with fixed and variable resetting delays of reinforcement in male and female Wistar rats. Psychology and Behavior, 52, 767-772.

Wilkenfield, J., Nickel, M., Blakely, E., \& Poling, A. (1992). Acquisition of lever-press responding in rats with delayed reinforcement: A comparison of three procedures. Journal of the Experimental Analysis of Behavior, 58, 431-443.

Williams, B. A. (1976). The effects of unsignaled delayed reinforcement. Journal of the Experimental Analysis of Behavior, 26, 441-449.

Zeiler, M. D. (1968). Fixed and variable schedules of responseindependent reinforcement. Journal of the Experimental Analysis of Behavior, 11, 405-414.

Recebido: 29/01/2007

Última revisão: 10/12/2007 Aceite final: 18/12/2007

\section{Sobre os autores:}

Cristina Moreira Fonseca: Doutora em Psicologia Experimental pelo Instituto de Psicologia - USP.

Gerson Yukio Tomanari: Doutor em Psicologia Experimental, Docente e Pesquisador do Instituto de Psicologia - USP

Endereço para correspondência: Prof. Gerson Yukio Tomanari - Universidade de São Paulo - Instituto de Psicologia - Av. Prof. Mello Moraes, 17 - 05508-030 - São Paulo/SP. Endereço eletrônico: tomanari@usp.br 